\title{
PERIODIC SOLUTIONS OF HIGH ACCURACY TO THE FORCED DUFFING EQUATION: PERTURBATION SERIES IN THE FORCING AMPLITUDE
}

\author{
LAWRENCE K. FORBES
}

(Received 13 February 1986)

\begin{abstract}
"Steady state" periodic solutions are sought to the forced Duffing equation. The solutions are expressed as formal Fourier series, giving rise to an infinte system of non-linear algebraic equations for the Fourier coefficients. This system is then solved using perturbation series in the amplitude of the forcing term. Solution profiles of high accuracy and phase-plane orbits are presented. The existence of limiting values of the forcing amplitude is discussed, and points of non-linear resonance are identified.
\end{abstract}

\section{Introduction}

In this paper, periodic solutions are sought to the forced Duffing equation

$$
d^{2} x / d \tau^{2}+A x+B x^{3}=F \cos \Omega \tau,
$$

in which $A, B, F$ and $\Omega$ are real constants, and $A>0$.

The deceptively simple ordinary differential equation (1.1) has been the subject of much research over many years, and arises naturally in a variety of physical contexts. For example, it is the governing equation for electrical circuits containing certain non-linear circuit components, it describes the forced oscillation of a spring-mass system in which the restoring force is no longer Hookean, and it also serves as an approximation to the behaviour of forced mechanical systems containing geometrical non-linearities (Davis [4], Arnold and Case [2]).

\footnotetext{
${ }^{1}$ Department of Mathematics, University of Queensland, St. Lucia, Queensland 4067, Australia. (1) Copyright Australian Mathematical Society 1987, Serial-fee code 0334-2700/87
} 
This paper is concerned with periodic "steady state" solutions to equation (1.1), where the frequency of the periodic solution is equal to the forcing frequency $\Omega$. The change of variable $t=\Omega \tau$ transforms equation (1.1) to

$$
\ddot{x}+\alpha x+\beta x^{3}=K \cos t,
$$

where dots denote differentiation with respect to $t$, and the new constants are $\alpha=A \Omega^{-2}, \beta=B \Omega^{-2}$ and $K=F \Omega^{-2}$. Equation (1.2) is supplemented with the periodic boundary conditions

$$
\begin{aligned}
& x(-\pi)=x(\pi), \\
& \dot{x}(-\pi)=\dot{x}(\pi) .
\end{aligned}
$$

The computation of periodic solutions to the forced Duffing equation (1.2) has been addressed by numerous authors. Davis [4], for example, expresses the solution in Fourier-series form, and obtains a truncated system of equations for the first few Fourier coefficients, which he solves graphically. His procedure has been extended somewhat by Bazley and Miletta [3] who present the full infinite system of equations for the Fourier coefficients. Urabe [10] anticipates that the Fourier coefficients might be found to high order using a digital computer, and it is a technique of this type which is employed in the present investigation. Here, however, we also express each Fourier coefficient as a perturbation series in $K$; details of this formulation may be found in Section 2. Our solution technique is thus similar in many respects to that adopted by Andersen and Geer [1] in their investigation of the van der Pol equation, and much use will be made of series-improvement devices of the type reviewed by van Dyke [11]. More recent research (e.g. Schmitt [8], Parlitz and Lauterborn [7] and Holmes [6]) has shown an extraordinary degree of complexity in the solutions of the forced Duffing equation, associated with the existence of bifurcation points, strange attractors and chaotic behaviour, and recourse is frequently made to advanced topological and functional-analytic arguments. These complicated phenomena will not be considered explicitly in the present paper, however.

\section{The Fourier- and Perturbation-series solution}

A solution is sought to the forced Duffing equation (1.2) in the Fourier-series form

$$
x(t)=\sum_{j=0}^{\infty} A_{2 \jmath+1} \cos ((2 j+1) t),
$$

which satisfies the boundary conditions (1.3) identically. It is convenient at this stage to form the intermediate product

$$
x^{2}(t)=\sum_{j=0}^{\infty} F_{2 j} \cos (2 j t)
$$


in which

$$
F_{0}=\frac{1}{2} \sum_{s=0}^{\infty}\left(A_{2 s+1}\right)^{2}
$$

and

$$
F_{2 J}=\sum_{s=0}^{\infty} A_{2 s+2 J+1} A_{2 s+1}+\frac{1}{2} \sum_{s=0}^{j-1} A_{2 s+1} A_{2 j-2 s-1}, j=1,2,3, \ldots .
$$

Equations (2.1) and (2.2) are now substituted into the Duffing equation (1.2) to yield an infinite system of algebraic equations for the Fourier coefficients $A_{1}, A_{3}, A_{5}, \ldots$. For ease of future reference, we shall present the full system of equations here. It is

$$
\begin{array}{r}
(\alpha-1) A_{1}+\frac{1}{2} \beta \sum_{s=0}^{\infty} F_{2 s+2} A_{2 s+1}+\frac{1}{2} \beta \sum_{s=0}^{\infty} F_{2 s} A_{2 s+1}+\frac{1}{2} \beta F_{0} A_{1}=K, \\
\text { for } j=0,
\end{array}
$$

and

$$
\begin{aligned}
& {\left[\alpha-(2 j+1)^{2}\right] A_{2 \jmath+1}+\frac{1}{2} \beta \sum_{s=0}^{\infty} F_{2 s+2 \jmath+2} A_{2 s+1}} \\
& +\frac{1}{2} \beta \sum_{s=0}^{\infty} F_{2 s} A_{2 s+2 \jmath+1}+\frac{1}{2} \beta \sum_{s=0}^{j} F_{2 s} A_{2 \jmath-2 s+1}=0, \\
& \quad \text { for } j=1,2,3, \ldots .
\end{aligned}
$$

Clearly the analytic solution of equations (2.3) cannot proceed without some further simplifying assumption. Several of these are available, but the simplest is to assume the perturbation series

$$
A_{2 j+1}=K^{2 j+1} \sum_{m=0}^{\infty} A_{2 j+1,2 m} K^{2 m}, \quad j=0,1,2, \ldots
$$

for each Fourier coefficient in equation (2.1). A similar expansion of the form

$$
F_{2 j}=K^{2 j} \sum_{m=0}^{\infty} F_{2 j, 2 m} K^{2 m}
$$

is required for the coefficients of the intermediate product (2.2a).

When the expressions (2.4) are substituted into equations (2.3), a system of recurrence relations is obtained, which can be solved to arbitrarily high order on a digital computer. For $j=m=0$, one finds $F_{00}=0$ and $A_{10}=(\alpha-1)^{-1}$; if 
$\beta=0$, these are the only non-zero coefficients, which gives the solution

$$
x(t)=\frac{K \cos t}{\alpha-1} \quad \text { for } \beta=0
$$

to the linear equation.

The full system of recurrence relations is too lengthy to be presented here, although fồ $m=\hat{\theta}$, oñe finuts the comparatively simple expression

$$
A_{2 \jmath+1,0}=-\frac{\beta}{2\left[\alpha-(2 j+1)^{2}\right]} \sum_{s=1}^{J} F_{2 s, 0} A_{2 \jmath-2 s+1,0},
$$

in which

$$
F_{2 \jmath, 0}=\frac{1}{2} \sum_{s=0}^{j-1} A_{2 s+1,0} A_{2,-2 s-1,0}, \quad j=1,2,3, \ldots
$$

Equations (2.5) and (2.6) indicate that the perturbation series (2.4) fails whenever $\alpha$ equals one of the eigenvalues $(2 j+1)^{2}, j=0,1,2, \ldots$, of the linear operator $(\beta=0)$.

The expansions for the first few Fourier coefficients, computed by hand, are as follows:

$$
\begin{aligned}
& A_{1}=K\left[\frac{1}{\alpha-1}-\frac{3 \beta K^{2}}{4(\alpha-1)^{4}}+\frac{3(5 \alpha-41) \beta^{2} K^{4}}{8(\alpha-9)(\alpha-1)^{7}}+0\left(K^{6}\right)\right] \\
& A_{3}=K^{3}\left[-\frac{\beta}{4(\alpha-9)(\alpha-1)^{3}}+\frac{3(5 \alpha-29) \beta^{2} K^{2}}{16(\alpha-9)^{2}(\alpha-1)^{6}}+0\left(K^{4}\right)\right] \\
& A_{5}=K^{5}\left[\frac{3 \beta^{2}}{16(\alpha-25)(\alpha-9)(\alpha-1)^{5}}+0\left(K^{2}\right)\right] \\
& A_{7}=K^{7}\left[-\frac{3(\alpha-13) \beta^{3}}{16(\alpha-49)(\alpha-25)(\alpha-9)^{2}(\alpha-1)^{7}}+0\left(K^{2}\right)\right] \\
& A_{9}=0\left(K^{9}\right)
\end{aligned}
$$

Again, the failure of the perturbation series (2.4) at the eigenvalues of the linear problem is evident. Clearly the assumed form of the perturbation series (2.4) is inadequate at these special values of $\alpha$, and a more general form of perturbation expansion is needed. For example, when $\alpha=9$, a solution may be obtained by allowing both $A_{1}$ and $A_{3}$ to be $O(K)$, and $A_{5}, A_{7}, A_{9}$ to be $O\left(K^{3}\right)$. After considerable calculation, a single solution is obtained at the resonance value 
$\alpha=9$. It is

$$
\begin{aligned}
& A_{1}=\frac{1}{8} K-\frac{3 \beta}{128}\left(\frac{1}{128}+\frac{\theta}{16}+\theta^{2}\right) K^{3}+0\left(K^{4}\right), \\
& A_{3}=\theta K+0\left(K^{2}\right) \\
& A_{5}=\frac{3 \beta}{512}\left(\frac{\theta}{8}+\theta^{2}\right) K^{3}+0\left(K^{4}\right), \\
& A_{7}=\frac{3 \beta}{1280} \theta^{2} K^{3}+0\left(K^{4}\right), \\
& A_{9}=\frac{\beta}{288} \theta^{3} K^{3}+0\left(K^{4}\right), \\
& A_{11}=0\left(K^{4}\right), \text { at } \alpha=9,
\end{aligned}
$$

where the constant $\theta$ is the real root of the cubic equation

$$
3 \theta^{3}+\frac{3}{32} \theta+\frac{1}{512}=0 .
$$

The system of recurrence relations derived from equations (2.3), of which equation (2.6) forms a part, has been programmed in the FORTRAN language on an IBM 3083E computer. To avoid the coefficients $A_{2,+1,2 m}$ becoming excessively small, with consequent computer underflow problems, the perturbation series (2.4) are re-written in terms of the new parameter $\varepsilon$ rather than $K$, where $\varepsilon=K / S_{c}$, and $S_{c}$ is an appropriate scale factor. Convenient values for $S_{c}$ are typically small integral multiples of $\alpha-1$. The only change that this entails is that $A_{10}$ must be replaced by $S_{c}(\alpha-1)^{-1}$ in the computer programme. To begin the computation, equations (2.6) are solved separately to some desired order $N$, giving $A_{10}, A_{30}, \ldots, A_{2 N+1,0}$. The first pass through the recurrence relations yields the coefficients $A_{12}, A_{32}, \ldots, A_{2 N-1,2}$, and so on until a triangular array of coefficients has been obtained. With $N=61$, the process of obtaining the coefficients requires about 150 seconds of CPU time.

Once the coefficients have been determined, the series (2.1) and (2.4) are combined and re-written in the form

$$
\begin{aligned}
x(t) & =\sum_{j=0}^{\infty} \varepsilon^{2 j+1} \sum_{m=0}^{\infty} A_{2 j+1,2 m} \varepsilon^{2 m} \cos ((2 j+1) t) \\
& \equiv \varepsilon \sum_{i=0}^{\infty} a_{i}(t) \varepsilon^{2 i}
\end{aligned}
$$


where $\varepsilon=K / S_{c}$ as above, and

$$
a_{\imath}(t)=\sum_{q=0}^{1} A_{2 q+1,2 i-2 q} \cos ((2 q+1) t) .
$$

The form (2.7) ensures optimum use of our triangular array of coefficients. In addition, equation (2.7) can easiliy be diffierentiated to give similar series for $\dot{x}(t)$ and $\ddot{x}(t)$. The series (2.7) is summed by recasting it as an $[m / n]$ Padé fraction, which is a rational function in $\varepsilon^{2}$ of the form

$$
\frac{x}{\varepsilon}=\frac{c_{0}+c_{1} \varepsilon^{2}+c_{2} \varepsilon^{4}+\cdots+c_{m} \varepsilon^{2 m}}{1+d_{1} \varepsilon^{2}+d_{2} \varepsilon^{4}+\cdots+d_{n} \varepsilon^{2 n}}+0\left(\varepsilon^{2 m+2 n+2}\right)
$$

in which the constants $c_{0}, \ldots, c_{m}$ and $d_{1}, \ldots, d_{n}$ are chosen so that the Taylorseries expansion of (2.8) agrees with equation (2.7) to order $2 N=2(m+n)$. In practice, Wynn's [12] epsilon algorithm is used to form the Pade approximants. As output, the computer program gives values of $t, x, \dot{x}, \ddot{x}$ and the residual

$$
\ddot{x}+\alpha x+\beta x^{3}-K \cos t .
$$

This last quantity provides a valuable check on the correctness of the recurrence relations, as well as the process of analytic continuation of the series (2.7) using Padé approximants, and indicates that the solutions often have accuracy in excess of eight significant figures.

\section{Presentation of Results}

\subsection{The hardening spring case $(\beta>0)$}

We consider first the case of the "hardening spring", $\beta>0$, so named because in a model of large-amplitude oscillation of a mass attached to a spring, the non-linear term $\beta x^{3}$ represents an additional restoring force beyond the usual Hooke's Law.

Figure 1 shows the solution $x$ as a function of $t$ for a single period $-\pi \leqslant t \leqslant \pi$, for the case $\alpha=5, \beta=1$, and the two different values of the forcing amplitude $K=4$ and $K=7$. When $K=4$ the profile is roughly cosinusoidal, as expected from the linearized solution (2.5) valid for suitably small $K$. For $K=7$, however, extra inflection points become apparent in the solution profile. 


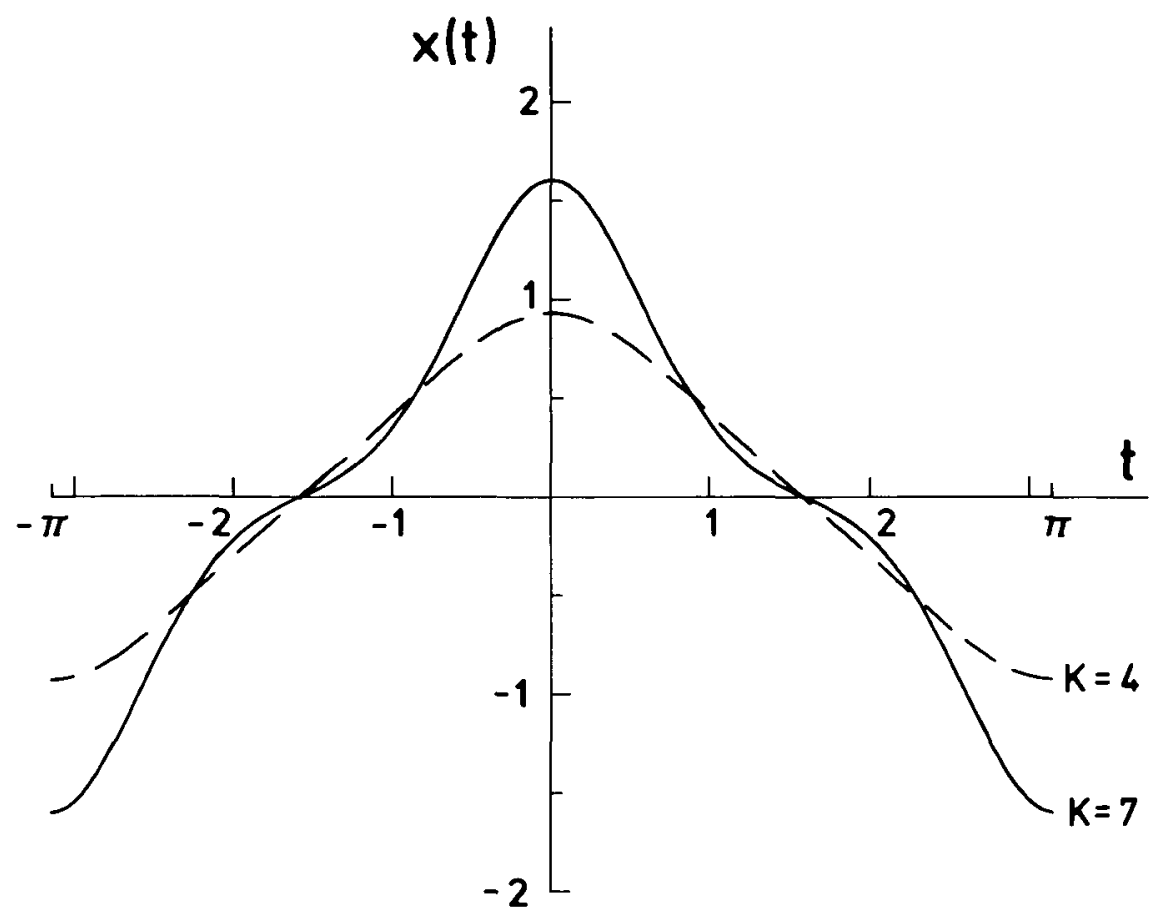

Figure 1. Solution profiles for $\alpha=5, \beta=1$ and amplitudes $K=4,7$.

The extent to which non-linearity affects the solution is made most evident in Figure 2, which is a sketch of the orbits in the phase plane for the same values of the parameters as in Figure 1. In the phase plane, the linearized solution (2.5) describes circular orbits of radius $K(\alpha-1)^{-1}$ centred at the origin, so that the departure from circularity of the orbits in Figure 2 is a direct measure of the effects of non-linearity. Even when $K=4$, the orbit is significantly distorted into a roughly elliptical shape, whilst for $K=7$ the orbit pinches in sharply at $x=0$.

For the case $\alpha=5, \beta=1$ investigated in Figures 1 and 2 it was found that the Padé fractions used to sum the series (2.7) suddenly failed to converge at about $K=7.3$. Accordingly, we seek the radius of convergence of the series

$$
\frac{x(0)}{\varepsilon}=\sum_{i=0}^{\infty} a_{i}^{(0)} \varepsilon^{2 i}
$$

in which

$$
a_{i}^{(0)}=\sum_{q=0}^{l} A_{2 q+1,2 i-2 q} .
$$




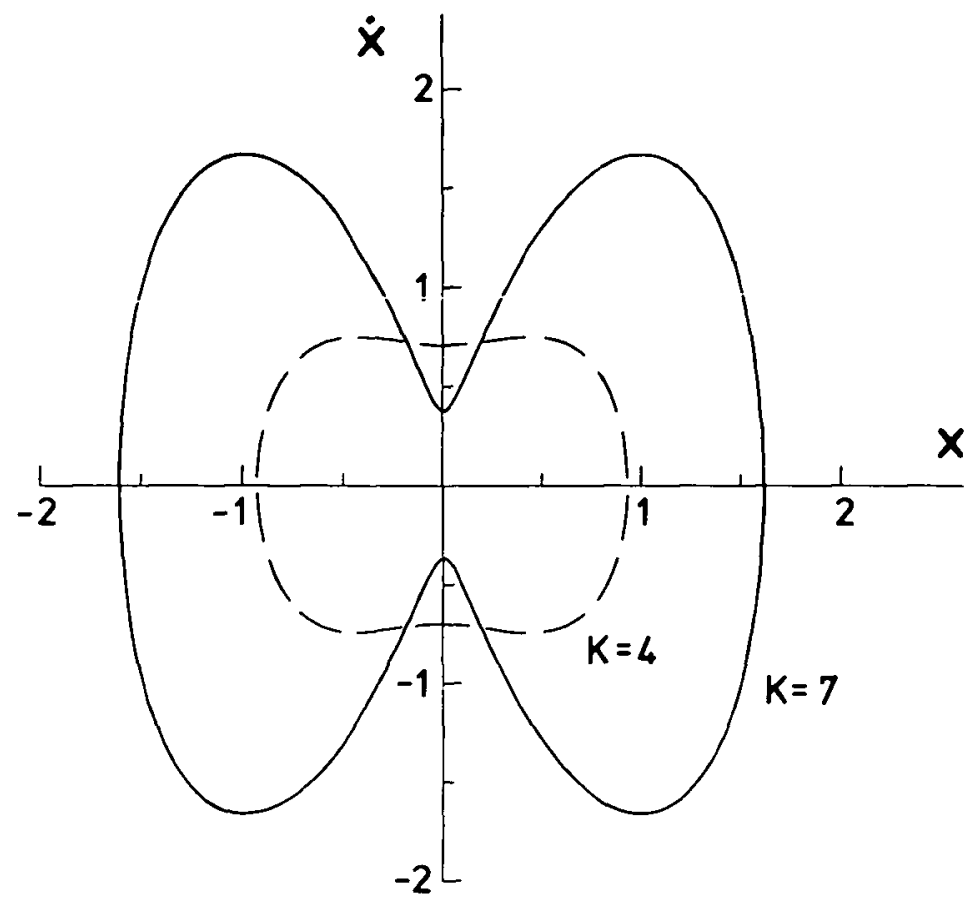

Figure 2. Phase-plane orbits for $\alpha=5, \beta=1$ and amplitudes $K=4,7$.

This is done by the method of Domb and Sykes [5]. Thus, if the nearest singularity of equation (3.1) is assumed to be of the form

$$
\frac{x(0)}{\varepsilon} \rightarrow \begin{cases}C\left(\varepsilon^{2}+\xi\right)^{\delta}, & \delta \neq 0,1,2, \ldots \\ C\left(\varepsilon^{2}+\xi\right)^{\delta} \log \left(\varepsilon^{2}+\xi\right), & \delta=0,1,2, \ldots\end{cases}
$$

where $C, \xi$ and $\delta$ are constants, then it follows (from the binomial theorem in the first instance) that

$$
a_{i}^{(0)} / a_{i-1}^{(0)} \rightarrow \frac{1}{\xi}\left(-1+\frac{1+\delta}{i}\right), \quad \text { as } i \rightarrow \infty
$$

The position of the singularity, $\varepsilon^{2}=-\xi$, and the singularity exponent $\delta$ may therefore be found from the limits

$$
-1 / \xi=\lim _{i \rightarrow \infty} a_{i}^{(0)} / a_{i-1}^{(0)}
$$


and

$$
\delta=\lim _{i \rightarrow \infty} \xi i(i+1)\left[a_{i}^{(0)} / a_{i-1}^{(0)}-a_{i+1}^{(0)} / a_{i}^{(0)}\right]-1 .
$$

These are estimated using the extrapolative $e_{1}$-transform due to Shanks [9].

When $\alpha=5$ and $\beta=1$, as in Figures 1 and 2, equation (3.2a) yields the nearest singularity at $K^{2}=-13.525$ and (3.2b) gives its exponent as $\delta=0.50$, which indicates that the singularity is of square-root type. In order to explain the failure of the Padé fractions at about $K=7.3$, we have mapped the singularity at $K^{2}=-13.525$ away to infinity using an Euler transform (see van Dyke [11]), in which the series (3.1) is re-expressed in terms of the new variable

$$
\hat{\varepsilon}=\varepsilon^{2} /\left(\varepsilon^{2}+\xi\right)
$$

with $\xi$ as given in equation (3.2a). The Domb-Sykes technique indicates that this new series has a singularity on the positive real axis of $K^{2}$ but is insufficiently accurate to give the precise value.

From the series (3.1) we have constructed the series for two new functions $\varepsilon^{2}(s)$ and $x(0) \varepsilon^{-1}(s)$, where $s$ is a pseudo-arclength defined by

$$
d s^{2}=\left[d\left(\varepsilon^{2}\right)\right]^{2}+[d(x(0) / \varepsilon)]^{2} .
$$

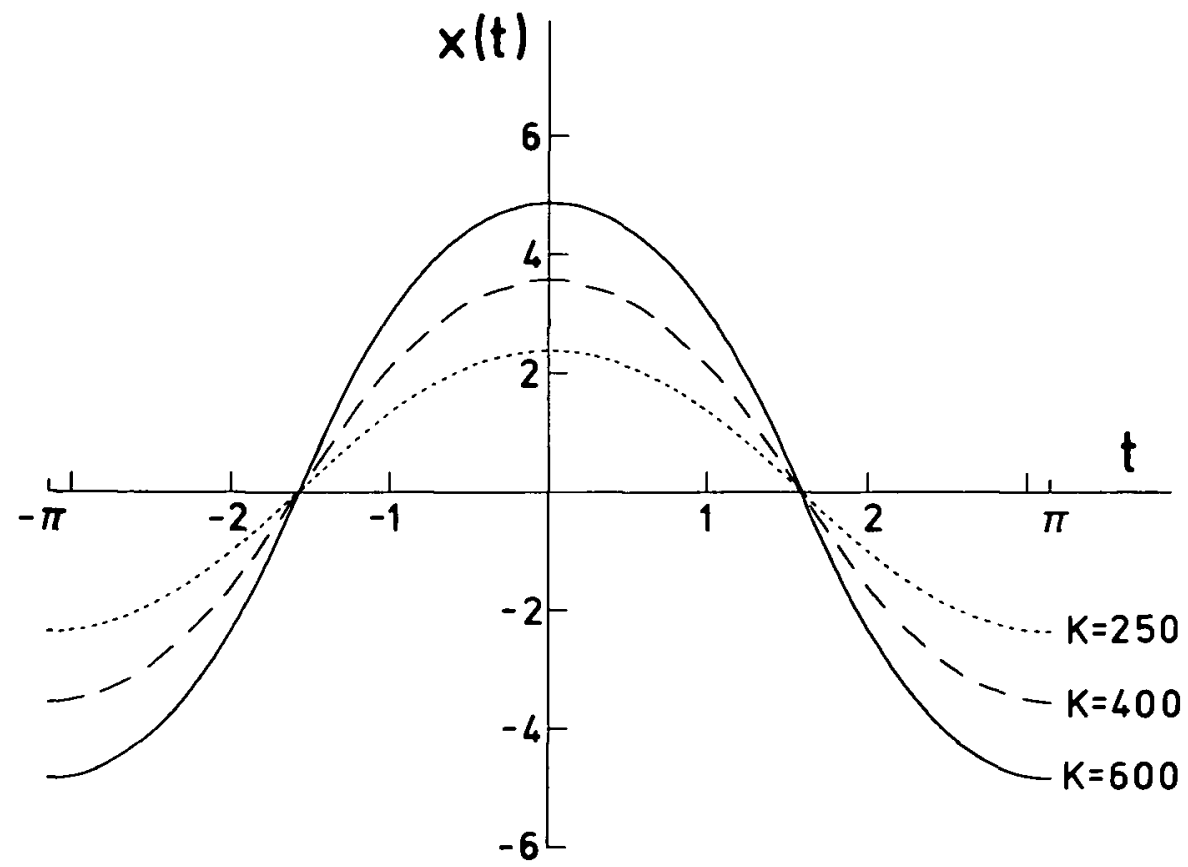

Figure 3. Solution profiles for $\alpha=101, \beta=1$ and amplitudes $K=250,400,600$. 
When these new series are summed using Padé approximants, they give the same result as before for $0<K<7.3$, but are able to yield highly converged values for $\varepsilon^{2}$ and $x(0) / \varepsilon$ with $K>7.3$. We therefore suggest that the failure of the original series (3.1) near $K=7.3$ is due to the presence of a pitchfork bifurcation at about this value of $K$.

Figure 3 shows solution profiles for the case $\alpha=101, \beta=1$ and the three values $K=250,400$ and 600 of the forcing amplitude. All tiree profiles appear to be roughly cosinusoidal, and to investigate the rôle played by non-linearity, it is again necessary to consider the solution behaviour in the phase plane.

In Figure 4, phase plane orbits are shown for the same values of the parameters as in Figure 3. When $K=250$, the orbit closely approximates the circle predicted by the linearized solution (2.5), but for $K=400$ and $K=600$ there are many subsidiary maxima and minima in each orbit.

The series (3.1) has again been examined for this case $\alpha=101, \beta=1$, and a graph of $x(0)$ against $K$ is given in Figure 5. The Padé fractions converge well up until about $K=624$, when they suddenly fail. Similarly, there is a narrow region around $K=405$ where the Padé fractions do not converge, and the shape of the graph in Figure 5 suggests a pole singularity near this value of $K$.

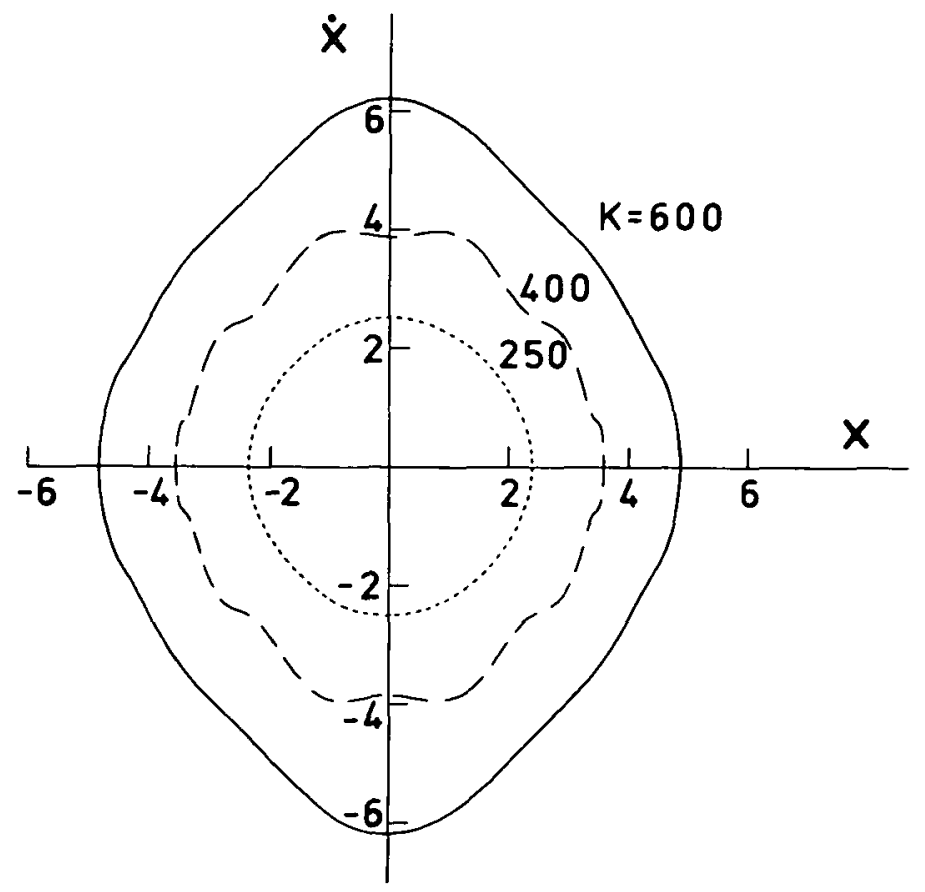

Figure 4. Phase-plane orbits for $\alpha=101, \beta=1$ and amplitudes $K=250,400,600$. 


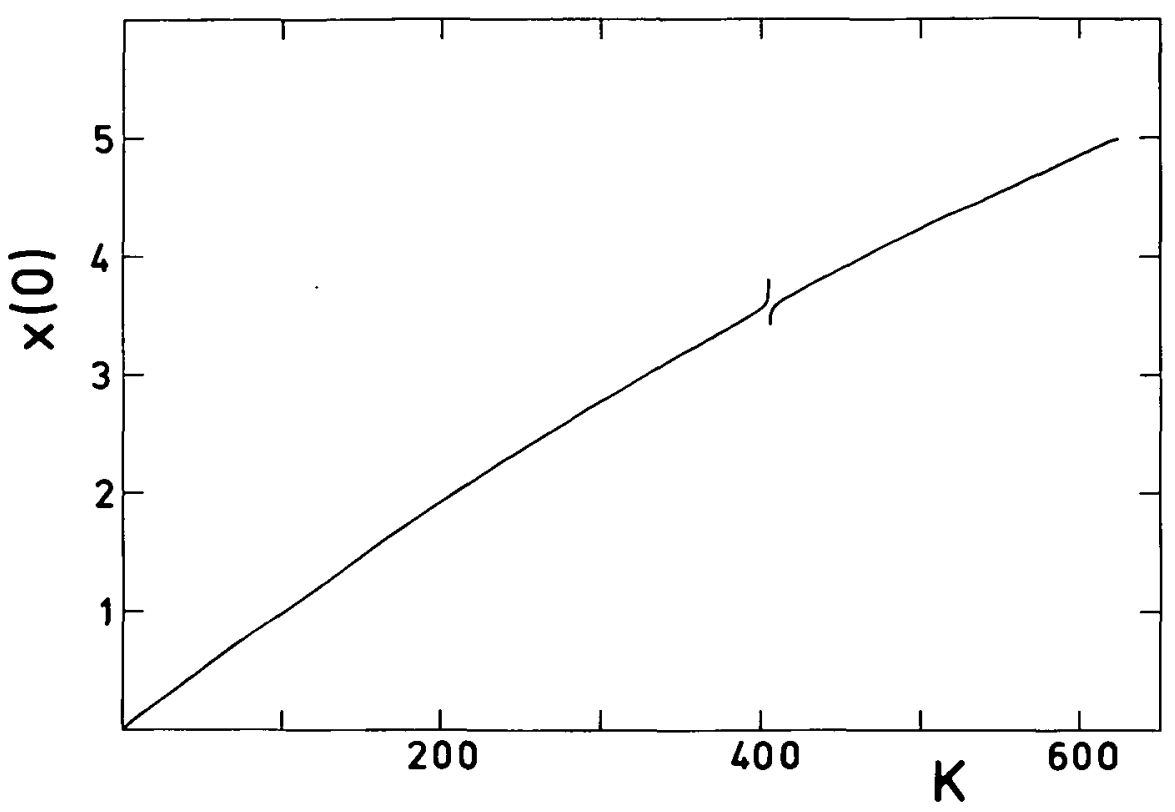

Figure 5. Solution amplitude $x(0)$ against forcing amplitude $K$ for $\alpha=101, \beta=1$.

The Domb-Sykes procedure, summarized in equations (3.2), is applied to the series (3.1) with $\alpha=101$ and $\beta=1$ and uncovers the existence of a square-root singularity again on the negative real axis of $K^{2}$, at $K^{2}=-104000$. This singularity is mapped away to infinity by the Euler transformation (3.3), and the new series now possesses a singularity at $K=404.93$, with $\delta=-1.0000$, as revealed by equations (3.2). Clearly this is a pole singularity, confirming the features in Figure 5, and presumably corresponds to a point of non-linear resonance in the physical system.

\subsection{The softening spring case $(\beta<0)$}

Figures 6 and 7 show, respectively, solution profiles and phase-plane orbits for $\alpha=5, \beta=-1$ and the two forcing amplitudes $K=2$ and $K=3.6$. The solution clearly becomes more like a square wave as $K$ is increased, and the corresponding phase-plane orbits become increasingly elongated in the vertical direction.

The Domb-Sykes procedure is applied to the series (3.1) in this case $\alpha=5$, $\beta=-1$, and shows that the nearest singularity now lies on the positive real axis of $K^{2}$, at $K=3.67$. Equation (3.2b) indicates that the singularity is of square-root type, with $\delta=0.50$. Such a singularity is the result of the solution surface folding back, and can be confirmed directly by reverting the series (3.1) to give a new 


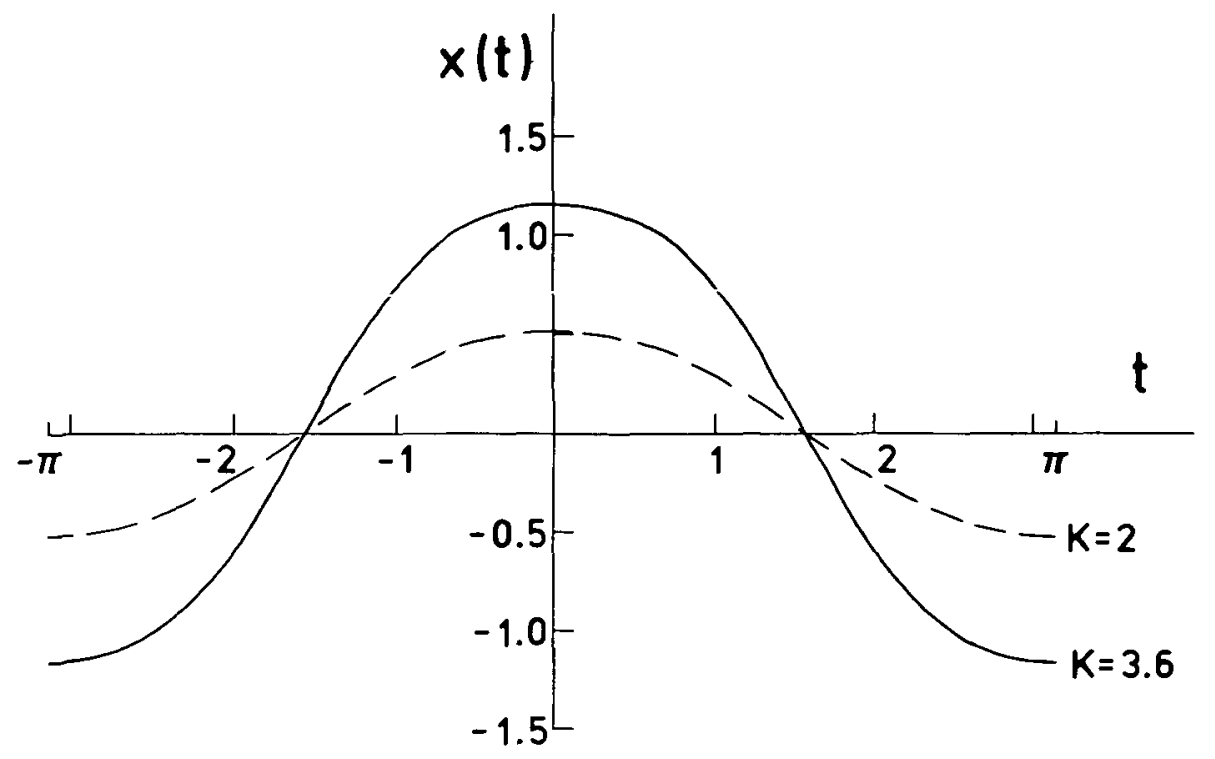

Figure 6. Solution profiles for $\alpha=5, \beta=-1$ and amplitudes $K=2,3.6$.

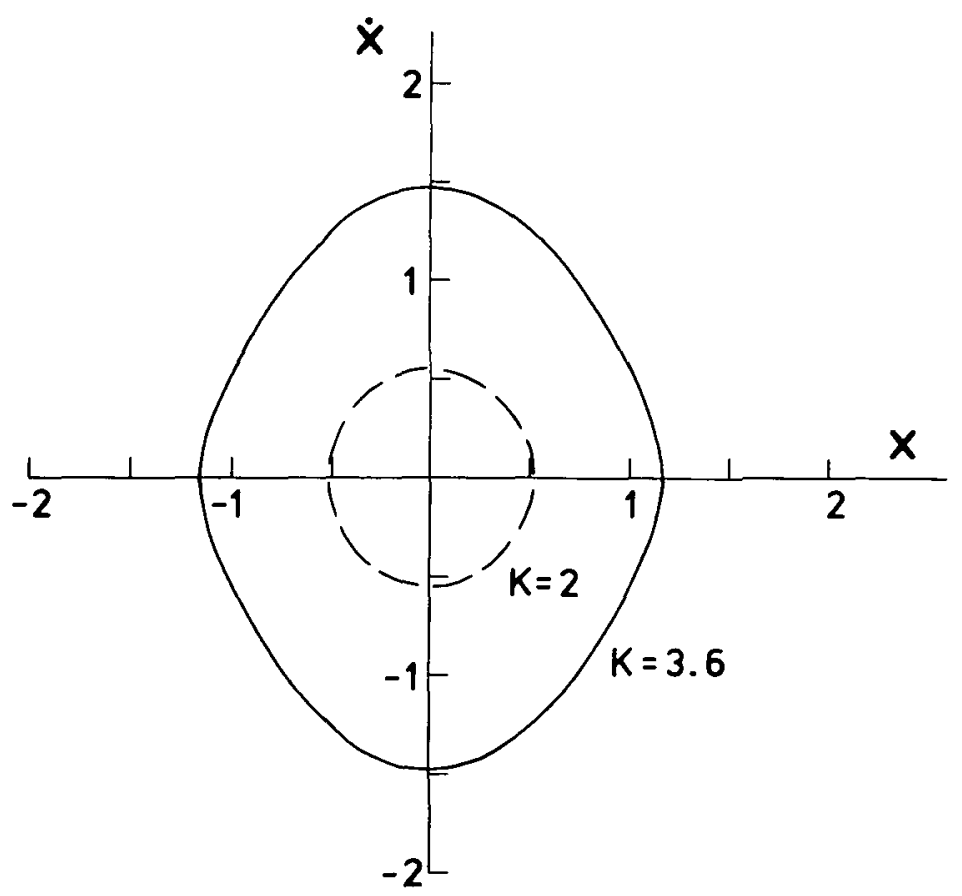

Figure 7. Phase-plane orbits for $\alpha=5, \beta=-1$ and amplitudes $K=2,3.6$. 
series of the form

$$
\varepsilon^{2}=\sum_{i=1}^{\infty} b_{i} \mu^{t}
$$

in which

$$
\mu=\left[x(0) / \varepsilon-a_{0}^{(0)}\right] / a_{1}^{(0)}
$$

and the coefficients $b_{i}$ are functions of $a_{j}^{(0)}, j=0,1, \ldots, i$. The new series (3.4) is summed using Padé approximants, as before.

In Figure 8 we present a graph of $x(0)$ against $K$ for the case $\alpha=5, \beta=-1$, obtained using both series (3.1) and (3.4). The reverted series (3.4) clearly indicates a fold at $K=3.67$, confirming the predictions of the Domb-Sykes technique. Multiple solutions are evidently a possible outcome at least within the narrow range of values $3.55<K<3.67$ of the forcing amplitude. The reverted series (3.4) fails suddenly at $K=3.55(x(0)=1.47)$, however, and it thus seems likely that a singularity exists near this point, although careful analysis of the various series presented here has so far failed to yield its exact nature or location.

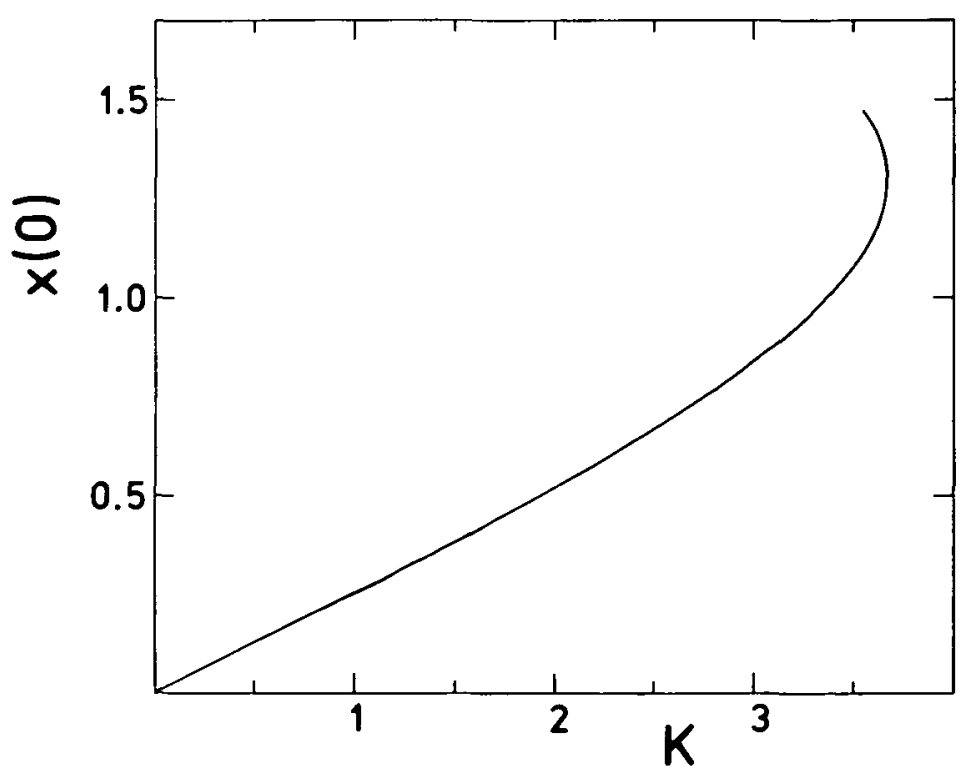

Figure 8. Solution amplitude $x(0)$ against forcing amplitude $K$ for $\alpha=5, \beta=-1$. 
In Figures 9 and 10, solution profiles and phase-plane orbits are shown for $\alpha=17, \beta=-1$ and the two values of forcing amplitude $K=16$ and $K=23$. The Domb-Sykes technique again indicates a square root singularity on the positive real axis of $K^{2}$, at $K \approx 23.4$, so that the solution at $K=23$ represents about the largest value of $K$ for which the present method is capable of giving accurate results. Figures 9 and 10 appear very similar to the results shown in Figures 1 and 2 , obtained with $\alpha=5$ and $\beta=1$, and it is perhaps possible that these two different branches of solution are somehow analytically linked.

The final case to be considered in this section is that obtained with $\alpha=101$ and $\beta=-1$. Three solution profiles are shown in Figure 11, with $K=200,300$ and 360. Whilst the profile with $K=200$ does not differ greatly from the predictions of linearized theory, that obtained with $K=360$ possesses numerous small wavelets "riding" on the overall solution profile and is thus strongly influenced by non-linearity.

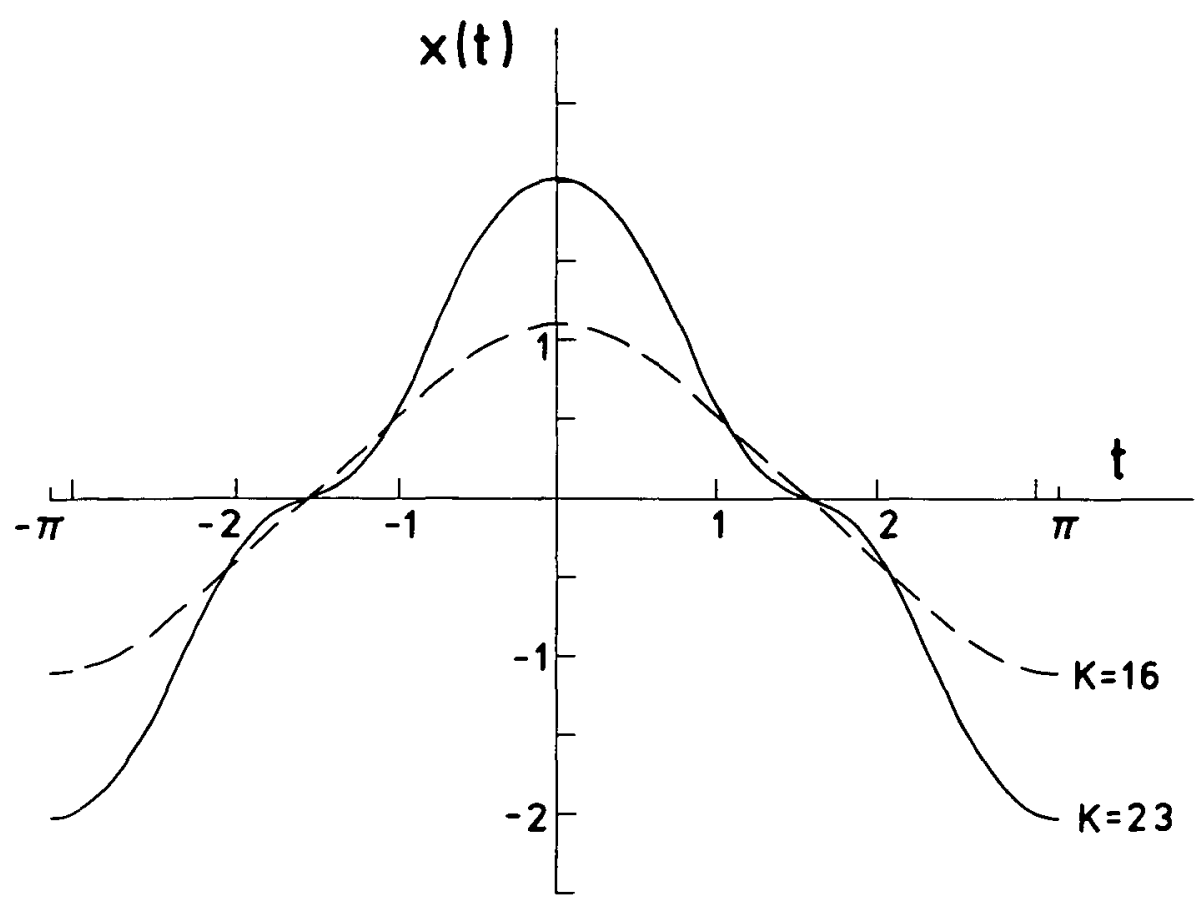

Figure 9. Solution profiles for $\alpha=17, \beta=-1$ and amplitudes $K=16,23$. 


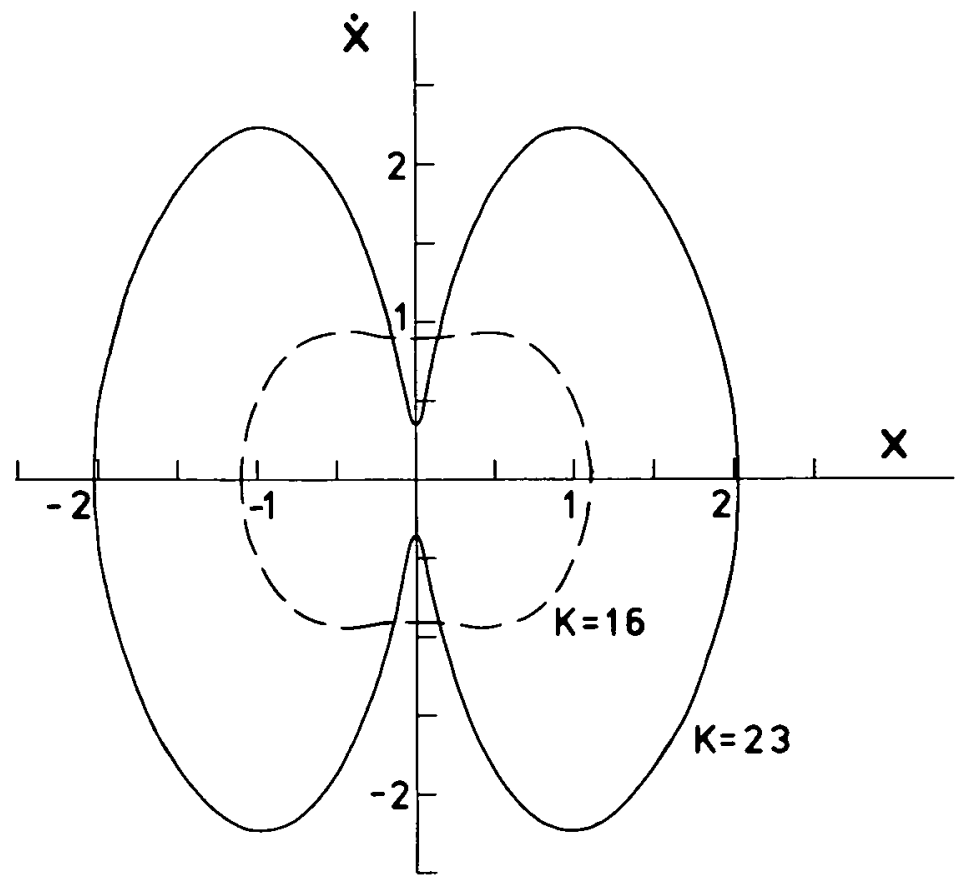

Figure 10. Phase-plane orbits for $\alpha=17, \beta=-1$ and amplitudes $K=16,23$.

Phase-plane orbits are shown in Figure 12 for $\alpha=101, \beta=-1$ and the same three values of $K$ as in Figure 11. Again it may be seen that the orbit obtained with $K=200$ differs but slightly from the circle predicted by linearized theory, whilst the exaggerated peaks and troughs of the orbit for $K=360$ indicate the strength of non-linear effects in this case.

Figure 13 shows a graph of $x(0)$ against $K$ for $\alpha=101, \beta=-1$. Unlike the results obtained with smaller $\alpha$, where the nearest singularity was of square-root type, the Domb-Sykes technique now indicates a pole singularity at about $K=322$. This is clearly visible in Figure 13 , and presumably corresponds to a point of non-linear resonance. The Padé fractions used to sum the series (3.1) fail again at about $K=386$, and the graph suggests that a square-root singularity may possibly occur at about this value of the forcing amplitude.

It is interesting to re-examine Figure 12 in the light of the information available from Figure 13. If one considers the portion of each orbit lying in the upper half of the phase plane, $\dot{x}>0$, it will be observed that this portion contains four upward-concave regions when $K=300$, and only three when $K=360$. In 


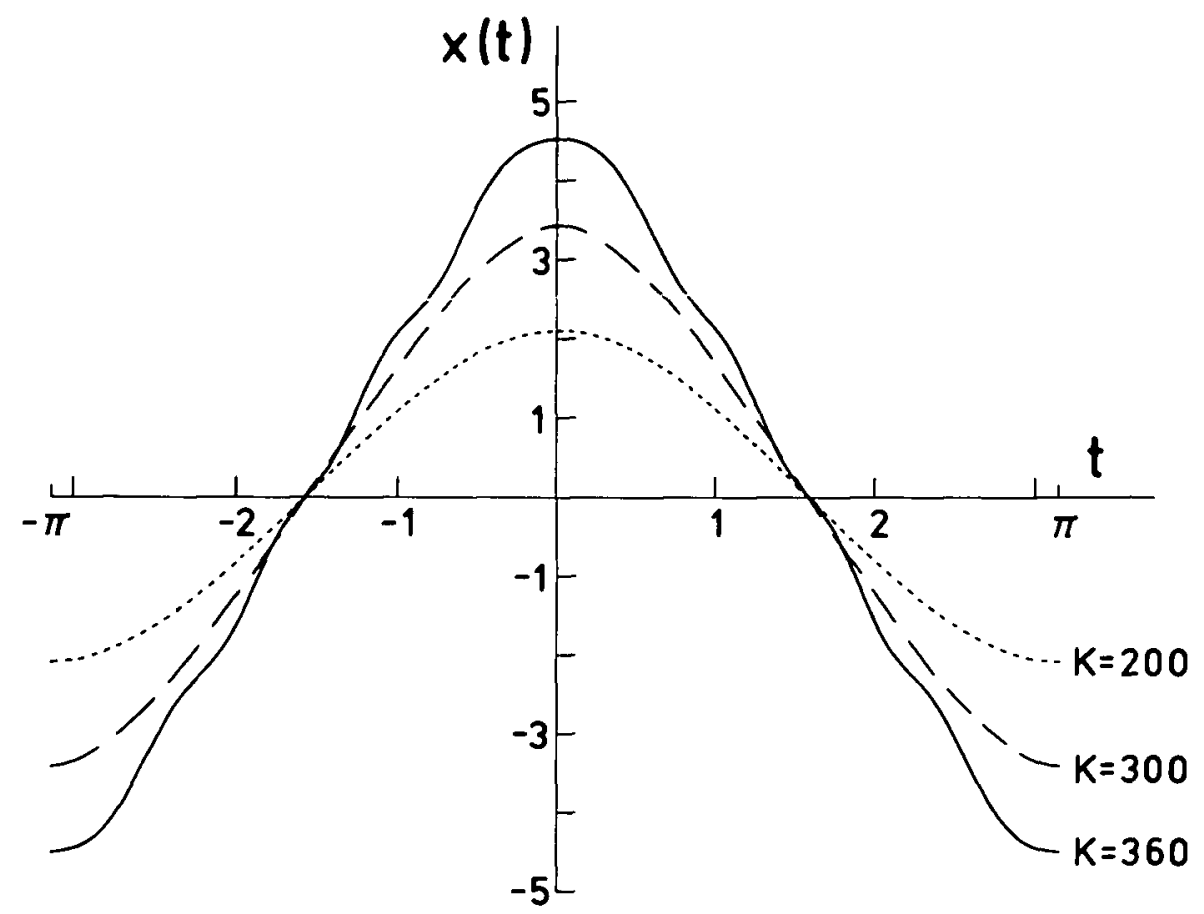

Figure 11. Solution profiles for $\alpha=101, \beta=-1$ and amplitudes $K=200,300,360$.

addition, the upper half of the orbit obtained with $K=300$ possesses a local maximum in $\dot{x}$ when $x=0$, whilst the orbit for $K=360$ possesses a local minimum at $x=0$. In fact, the orbit for $K=360$ appears qualitatively similar to those obtained with $\alpha$ lying between the singular values 49 and 81 , and we speculate that a different branch of solution has been entered upon crossing the point of non-linear resonance at $K=322$ in Figure 13.

The results presented in this paper for the case of the softening spring have all had the value $\beta=-1$ in common. Although the particular form of the series expansions (2.4) is not the most convenient for investigating the influence of $\beta$ upon the solution, we have run the computer programs for several different values of $\beta$ and find that there is little qualitative change in the solution profiles as a result of altering $\beta$ at a fixed value of $\alpha$, apart from a change of scale. 


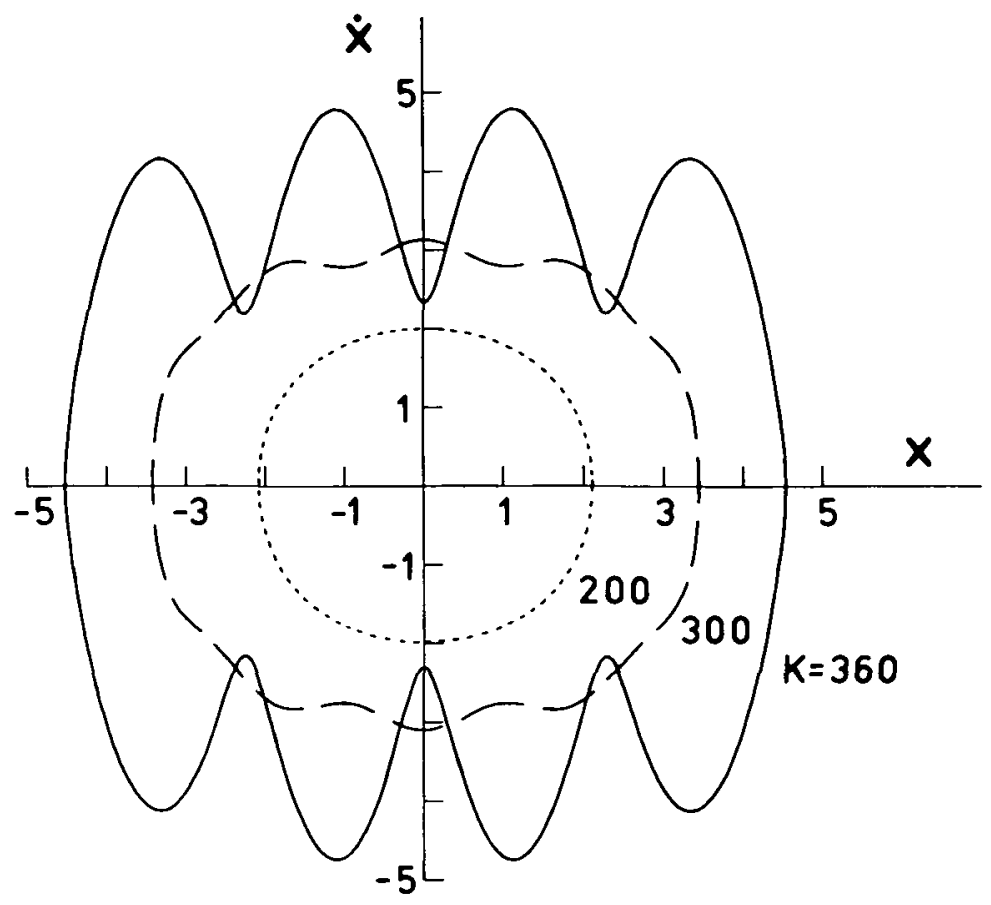

Figure 12. Phase-plane orbits for $\alpha=101, \beta \beta=-1$ and amplitudes $K=200,300,360$.

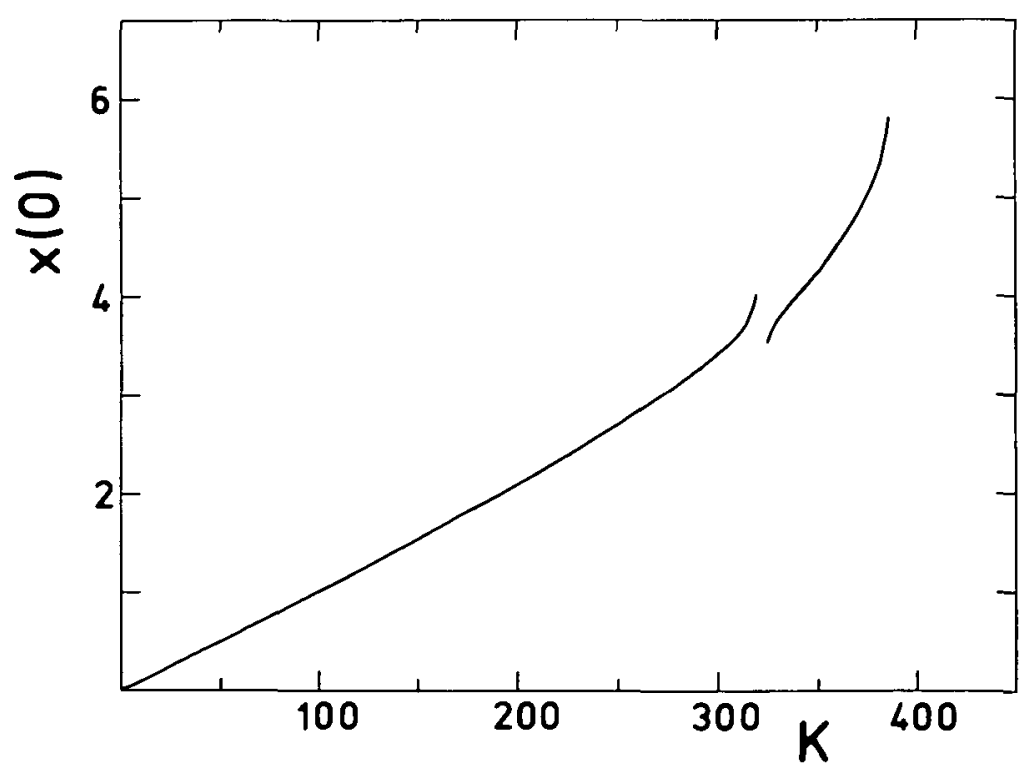

Figure 13. Solution amplitude $x(0)$ against forcing amplitude $K$ for $\alpha=101, \beta=-1$. 


\section{Summary}

Periodic solutions of high accuracy have been found for the forced Duffing equation. The solution technique is semi-analytical, and involves the use of Fourier series combined with perturbation expansions in the amplitude of the forcing term. The assumed form of the perturbation series fails to yield a solution whenever the coefficient of the linear term equals one of the eigenvalues of the linear problem, but between these singular values, the series converge to a unique solution. Limiting values for the forcing amplitude have been found in every case investigated, but the nature of these limits varies. In the case of the softening spring (when the coefficient of the non-linear term has negative sign) the limiting values of forcing amplitude appear to correspond to a fold in the solution surface, and the consequent possibility of multiple solutions. For the hardening spring case, when the sign of the non-linear term is positive, the nature of the limiting value of amplitude is less clear, but is possibly associated with a pitchfork bifurcation.

In both the hardening and softening spring cases, points of nonlinear resonance have been identified. It appears that a different branch of solution may be entered upon crossing such points. The stability of such branches, however, is not discussed here.

\section{References}

[1] C. M. Andersen and J. F. Geer, "Power series expansions for the frequency and period of the limit cycle of the van der Pol equation", SIAM J. Appl. Math. 42 (1982), 678-693.

[2] T. W. Arnold and W. Case, "Nonlinear effects in a simple mechanical system", Amer. J. Phys. 50 (1982), 220-224.

[3] N. W. Bazley and P. Miletta, "Approximations to periodic solutions of a Duffing equation", ZAMP 34 (1983), 301-309.

[4] H. T. Davis, Introduction to nonlinear differential and integral equations (Dover, New York, 1962).

[5] C. Domb and M. F. Sykes, "On the susceptibility of a ferromagnetic above the Curie point", Proc. Roy. Soc. London Ser. A 240 (1957), 214-228.

[6] P. Holmes, "A nonlinear oscillator with a strange attractor", Philos. Trans. Roy. Soc. London Ser A. 292 (1979), 418-448.

[7] U. Parlitz and W. Lauterborn, "Superstructure in the bifurcation set of the Duffing equation $\ddot{x}+d \dot{x}+x+x^{3}=f \cos (\omega t) "$, Phys. Lett. $A 107$ (1985), 351-355.

[8] B. V. Schmitt, "Sur la structure de l'equation de Duffing sans dissipation", SIAM J. Appl. Math. 42 (1982), 868-894.

[9] D. Shanks, "Non-linear transformations of divergent and slowly convergent sequences", $J$. Math. Phys. 34 (1955), 1-42.

[10] M. Urabe, "Galerkin's procedure for non-linear periodic systems", Arch. Rattonal Mech. Anal. 20 (1965), 120-152.

[11] M. D. van Dyke, "Analysis and improvement of perturbation series", Quart. J. Mech. Appl. Math. 27 (1974), 423-450.

[12] P. Wynn, "On the convergence and stability of the epsilon algorithm", SIAM J. Numer. Anal. 3 (1966), 91-121. 